\title{
Compact and Wideband CPW Wilkinson Power Dividers for GaN MMIC Applications
}

\author{
Batuhan Sutbas ${ }^{* \# 1}$, Ekmel Ozbay ${ }^{* \# 2}$, Abdullah Atalar ${ }^{\# 3}$ \\ *Nanotechnology Research Center (NANOTAM), Turkey \\ ${ }^{\#}$ Department of Electrical and Electronics Engineering, Bilkent University, Turkey \\ $\left\{{ }^{1}\right.$ sutbas, ${ }^{2}$ ekmel, ${ }^{3}$ atalar $\} @$ ee.bilkent.edu.tr
}

\begin{abstract}
This paper presents two types of modified CPW Wilkinson power dividers at $X$-band using GaN MMIC technology on a $\mathrm{SiC}$ substrate. Lumped element equivalents of the transmission line arms are used and they are capacitively loaded to achieve a reduced circuit size of $\lambda / 14 \times \lambda / 8$. A symmetrical series $R L C$ circuit in the isolation network is used to compensate for the bandwidth degradation after circuit miniaturization maintaining a fractional bandwidth of $29 \%$ for input/output return losses and isolation better than $20 \mathrm{~dB}$ with an extra insertion loss less than $0.35 \mathrm{~dB}$.
\end{abstract}

Keywords - coplanar waveguide, compact power divider, Wilkinson power divider, MMICs.

\section{INTRODUCTION}

The Wilkinson power divider is a symmetrical device commonly used in microwave engineering to divide the input signal into equiphase and equiamplitude output signals. It provides good port matching and high isolation between the output ports while maintaining a low loss operation [1]. Although the Wilkinson power divider is preferred for monolithic microwave integrated circuits (MMIC) due to its ease of fabrication and planar structure, it is not useful in wideband applications and consumes valuable chip area because of its quarter-wave length arms. In order to address the first issue and improve the bandwidth, multisection hybrids with a number of cascaded transmission line arms and bridging resistors [2] and a modified isolation network with a series $R L C$ circuitry [3], [4] have been proposed. Regarding the second issue to reduce the circuit size, lumped element $\pi$-network and T-network equivalents of the quarter-wave length arms [5], [6] and capacitive or inductive line loading [7], [8] have been suggested. On the one hand, techniques proposed for wideband operation require more circuit area; on the other hand, modifications suggested on the original divider to reduce its size decrease the bandwidth of operation.

In this work, two types of modified power dividers are proposed. Type-I is a miniaturization of the original Wilkinson power divider using lumped elements achieving a small circuit area. Type-II is a further miniaturized lumped element Wilkinson power divider utilizing capacitive loading at the ends of the divider arms while preserving operational bandwidth by having a symmetrical series $R L C$ circuitry in the isolation network. Analytical design equations of the component values for proposed dividers are presented. Dividers of both types are designed at $X$-band using coplanar waveguide (CPW) technology with practical design considerations. The measured input/output return loss, isolation and insertion loss parameters of the fabricated dividers are presented.

\section{ANALYSIS AND DESIGN}

\section{A. Type-I Power Divider}

Proposed network for the divider of type-I is depicted in Fig. 1. Quarter-wave transmission line arms of the Wilkinson power divider are replaced by their lumped element T-network equivalents. The bridging isolation network is composed of a resistor only. The required inductor and capacitor values are found by equating the $\mathrm{ABCD}$ parameters of the Wilkinson divider transmission line arm given in (1) and ABCD parameters of the lumped element T-network given in (2).

$$
\begin{gathered}
{\left[\begin{array}{ll}
A & B \\
C & D
\end{array}\right]=\left[\begin{array}{cc}
\cos \beta l & j Z_{c} \sin \beta l \\
j Y_{c} \sin \beta l & \cos \beta l
\end{array}\right]} \\
{\left[\begin{array}{ll}
A & B \\
C & D
\end{array}\right]=\left[\begin{array}{cc}
1-X_{L} B_{C} & j X_{L} \\
j X_{L}\left(2-X_{L} B_{C}\right) & 1-X_{L} B_{C}
\end{array}\right]}
\end{gathered}
$$

where

$$
\begin{gathered}
Z_{c}=\sqrt{2} Z_{0}, \quad \beta l=\pi / 2 \\
X_{L}=2 \pi f_{0} L_{T} \quad, \quad B_{C}=1 / 2 \pi f_{0} C_{T}
\end{gathered}
$$

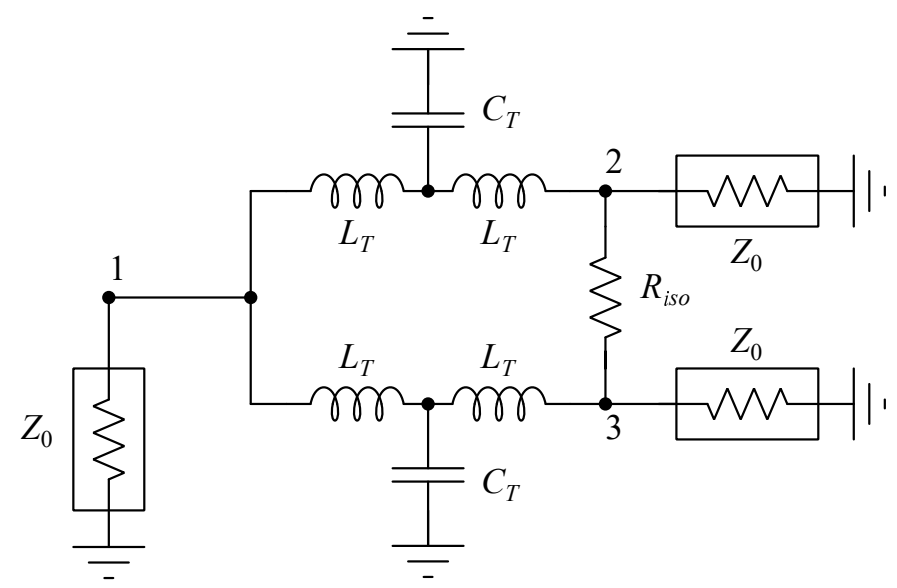

Fig. 1. Proposed network for type-I divider. 
Therefore, the required component values are

$$
\begin{gathered}
L_{T}=\frac{\sqrt{2} Z_{0}}{2 \pi f_{0}} \\
C_{T}=\frac{\sqrt{2}}{4 \pi f_{0} Z_{0}} \\
R_{\text {iso }}=2 Z_{0}
\end{gathered}
$$

where $f_{0}$ is the design center frequency and $Z_{0}$ is the characteristic impedance of the three ports. Following the described procedure, a divider at $X$-band is designed considering that the divider width must be less than the MMIC width and divider length is the critical dimension for total chip area. Short transmission lines are added at the input port and at the lumped element divider arms to improve bandwidth performance. Following the optimization process, shunt capacitors $\left(C_{T}\right)$ are eliminated due to parasitic capacitances of the inductors. Total circuit size is $1.14 \mathrm{~mm} \times 1.91 \mathrm{~mm}$ $(0.09 \lambda \times 0.14 \lambda)$. The implemented divider is shown in Fig. 2 .

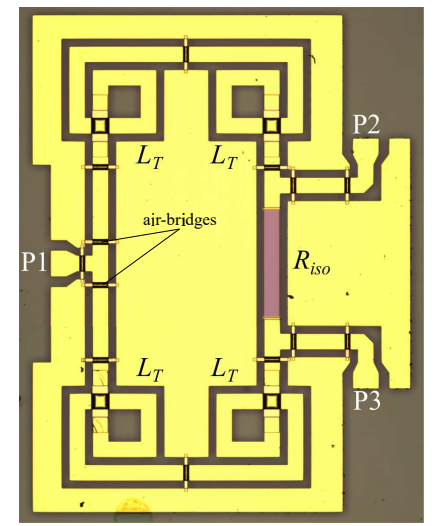

Fig. 2. Microscope image of the fabricated power divider of type-I.

\section{B. Type-II Power Divider}

In Fig. 3, the proposed network for the divider of type-II is presented. Shunt capacitors are added at the ends of the transmission line arms of the Wilkinson power divider to reduce their length. In order to compensate for the isolation bandwidth degradation a symmetrical series $R L C$ circuit is used in the isolation network instead of a single resistor. The inductor and capacitor in the isolation network resonate at the design center frequency. Considering symmetry of the network, even-odd mode analysis is carried out to find component values for perfect port matching at all ports and perfect isolation between output ports at the design center frequency [9].

$$
\begin{gathered}
C_{1}=\frac{\sqrt{2} \cos \beta l}{2 \pi f_{0} Z_{0}} \\
C_{2}=\frac{\sqrt{2} \cos \beta l}{4 \pi f_{0} Z_{0}} \\
L_{e q}=\frac{\sqrt{2} Z_{0}}{2 \pi f_{0}(1+\cos \beta l)}
\end{gathered}
$$

$$
\begin{gathered}
C_{e q}=\frac{\sqrt{2} \sin ^{2} \beta l}{4 \pi f_{0} Z_{0}} \\
R_{i s o}=2 Z_{0} \\
4 \pi^{2} f_{0}^{2} L_{i s o} C_{\text {iso }}=1
\end{gathered}
$$

where $f_{0}$ is the design center frequency, $\beta l$ is the electrical length of the reduced arm at the design center frequency, and $Z_{0}$ is the characteristic impedance of the system.

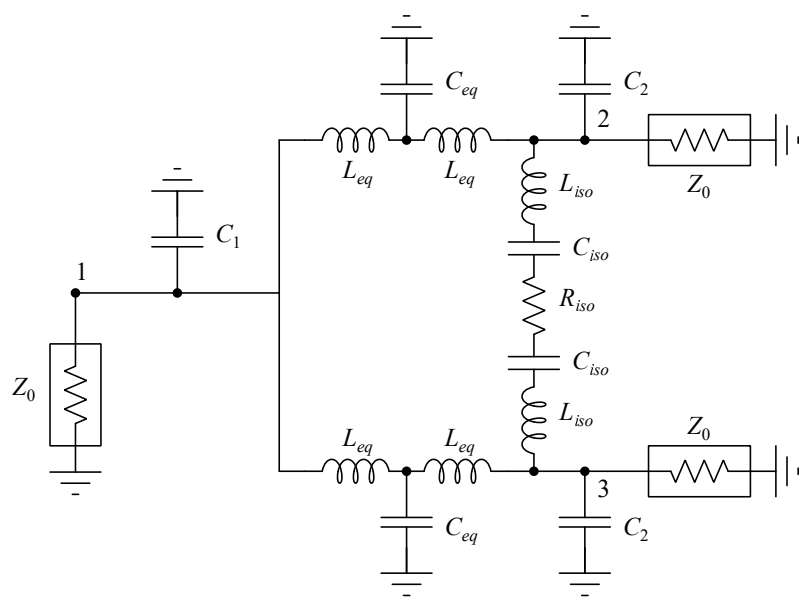

Fig. 3. Proposed network for type-II divider.

For the practical design case at $X$-band, isolation network is tuned for maximum bandwidth. Shunt capacitors $C_{e q}$ and $C_{2}$ are obtained by inductor parasitic and tee junction capacitances. Inductors in the isolation arm are realized by high impedance transmission lines. The additional circuitry in the isolation network not only compensates for the bandwidth, but also increases the distance between the output ports without additional loss. The output ports need to be separated anyway for physical isolation and practical use in MMICs. Total circuit size is $0.93 \mathrm{~mm} \times 1.66 \mathrm{~mm}(0.07 \lambda \times 0.13 \lambda)$ with $29 \%$ size reduction compared to the divider of type-I. The implemented divider is shown in Fig. 4.

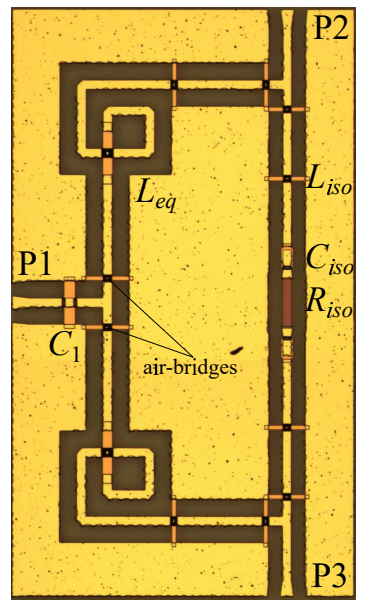

Fig. 4. Microscope image of the fabricated power divider of type-II. 


\section{FABRICATION AND EXPERIMENTAL RESULTS}

The modified Wilkinson power divider designs are fabricated with a $\mathrm{GaN}$ on $\mathrm{SiC}$ MMIC process with a substrate thickness of $100 \mu \mathrm{m}$ and dielectric constant of 9.7. Two layers of interconnection metals and air-bridges are formed using gold. Thin film resistors are made of $\mathrm{Ni}-\mathrm{Cr}$ with a sheet resistance of $15 \Omega / \square$. Metal-insulator-metal (MIM) capacitors are fabricated using $\mathrm{Si}_{x} \mathrm{~N}_{y}$ as an insulator with dielectric constant of 7.35. Quarter-wave length at $10 \mathrm{GHz}$ is approximately $3.3 \mathrm{~mm}$ for reference.

3 -port calibration and measurements are performed using Cascade $^{1}$ RF wafer probe station, GGB $^{2}$ GSG150 picoprobes and $R \& S^{3}$ ZVA40 vector network analyzer. Simulation results ${ }^{4}$ and measurement results of the dividers are in good agreement. Figs. 5-8 show the simulated and measured S-parameters of divider type-I. The measured input reflection and output reflection coefficients are less than $-20 \mathrm{~dB}$, the isolation is at least $20 \mathrm{~dB}$ and the excess insertion loss is less than $0.35 \mathrm{~dB}$ from $8.3 \mathrm{GHz}$ to $10.6 \mathrm{GHz}$ with a fractional bandwidth of $24 \%$. The amplitude imbalance and phase imbalance at the output ports are less than $0.1 \mathrm{~dB}$ and $0.8^{\circ}$, respectively. We measured three more power dividers and they show similar performance.

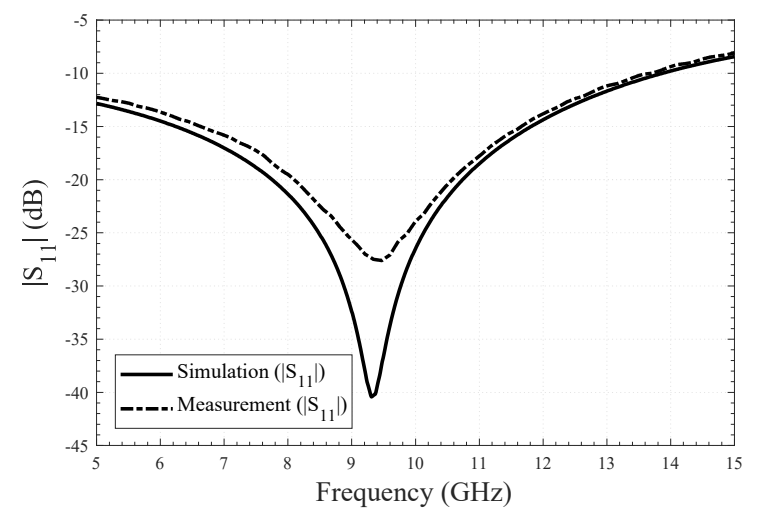

Fig. 5. Simulated and measured values of $\left|\mathrm{S}_{11}\right|$ of divider type-I as a function of frequency.

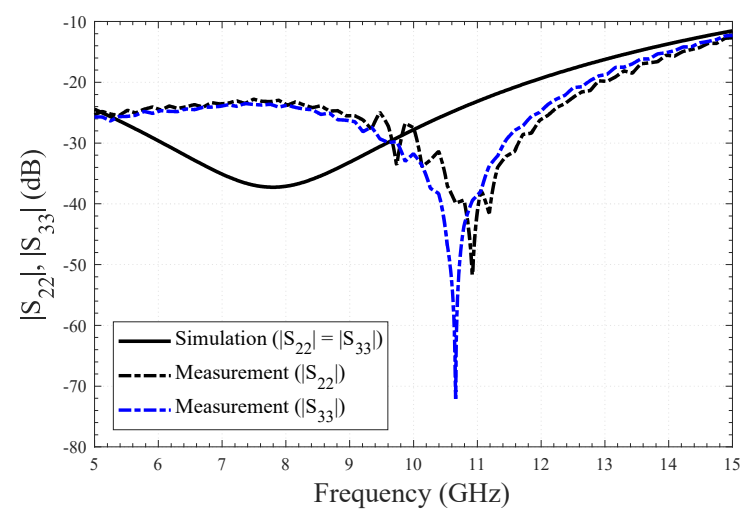

Fig. 6. Simulated and measured values of $\left|S_{22}\right|$ and $\left|S_{33}\right|$ of divider type-I as a function of frequency.

${ }^{1}$ Cascade Microtech, Inc., Beaverton, OR 97008, USA

${ }^{2}$ GGB Industries, Inc., Naples, FL 34104, USA

${ }^{3}$ Rohde \& Schwarz GmbH \& Co. KG, Munich, Germany

${ }^{4}$ ADS 2016.01, Keysight Technologies, Inc., Santa Rosa, CA 95403, USA

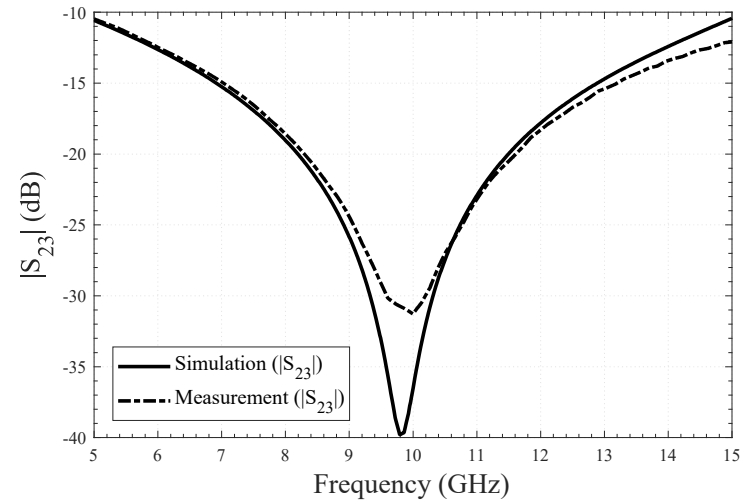

Fig. 7. Simulated and measured values of $\left|\mathrm{S}_{23}\right|$ of divider type-I as a function of frequency.

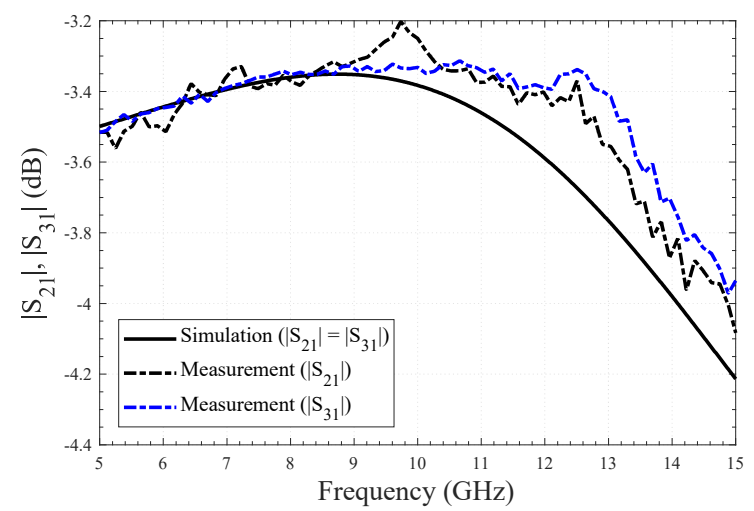

Fig. 8. Simulated and measured values of $\left|\mathrm{S}_{21}\right|$ and $\left|\mathrm{S}_{31}\right|$ of divider type-I as a function of frequency.

Figs. 9-12 compare the simulated and measured $\mathrm{S}$-parameters of divider type-II. According to the measurement results, the input and output return losses are better than $20 \mathrm{~dB}$, the isolation is at least $20 \mathrm{~dB}$ and the extra insertion loss is less than $0.35 \mathrm{~dB}$ from $8.9 \mathrm{GHz}$ to $11.9 \mathrm{GHz}$ with a fractional bandwidth of $29 \%$. The amplitude imbalance and phase imbalance at the output ports are less than $0.05 \mathrm{~dB}$ and $0.4^{\circ}$, respectively. Simulation and measurement results for both dividers have been summarized in Table 1 .

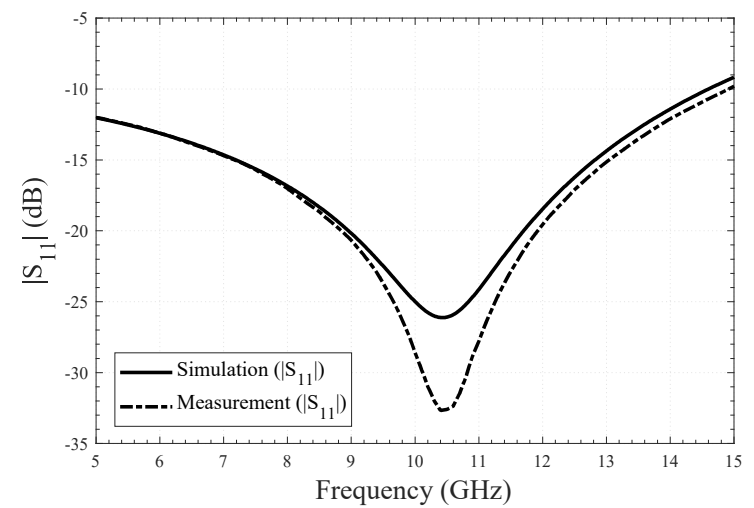

Fig. 9. Simulated and measured values of $\left|\mathrm{S}_{11}\right|$ of divider type-II as a function of frequency. 
Table 1. Simulated vs. measured input return loss, output return loss, isolation and insertion loss bandwidths for both types of power dividers.

\begin{tabular}{|c|c|c|c|c|c|c|c|c|c|c|}
\hline Performance Parameter & Input RL & $20 \mathrm{~dB})$ & Output RI & $(20 \mathrm{~dB})$ & Isolation $(2$ & $20 \mathrm{~dB})$ & Excess IL & $0.45 \mathrm{~dB})$ & Operation: & Region \\
\hline Divider Type & $\begin{array}{c}\text { BW } \\
\text { (GHz) }\end{array}$ & $\begin{array}{c}\text { FBW } \\
(\%)\end{array}$ & $\begin{array}{c}\text { BW } \\
(\mathbf{G H z})\end{array}$ & $\begin{array}{c}\text { FBW } \\
(\%)\end{array}$ & $\begin{array}{c}\text { BW } \\
(\mathbf{G H z})\end{array}$ & $\begin{array}{c}\text { FBW } \\
(\%)\end{array}$ & $\begin{array}{c}\text { BW } \\
(\mathbf{G H z})\end{array}$ & $\begin{array}{c}\text { FBW } \\
(\%)\end{array}$ & $\begin{array}{c}\text { BW } \\
(\mathbf{G H z})\end{array}$ & $\begin{array}{c}\text { FBW } \\
(\%)\end{array}$ \\
\hline Type-I Simulation & $7.8-10.7$ & 31 & $4.0-11.8$ & 99 & $8.2-11.5$ & 34 & $5.9-10.9$ & 60 & $8.2-10.7$ & 26 \\
\hline Type-I Measurement & $8.1-10.6$ & 27 & $3.5-12.8$ & 114 & $8.3-11.6$ & 33 & $6.2-12.8$ & 69 & $8.3-10.6$ & 24 \\
\hline Type-II Simulation & $9.0-11.7$ & 26 & $7.2-13.6$ & 62 & $8.3-12.4$ & 39 & $6.3-12.4$ & 65 & $9.0-11.7$ & 26 \\
\hline Type-II Measurement & $8.9-11.9$ & 29 & $8.5-16.3$ & 63 & $8.0-12.0$ & 40 & $6.1-13.2$ & 74 & $8.9-11.9$ & 29 \\
\hline
\end{tabular}

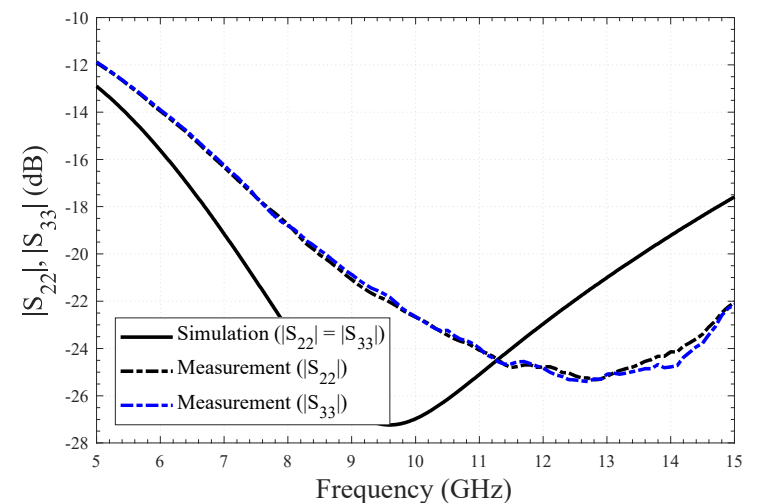

Fig. 10. Simulated and measured values of $\left|\mathrm{S}_{22}\right|$ and $\left|\mathrm{S}_{33}\right|$ of divider type-II as a function of frequency.

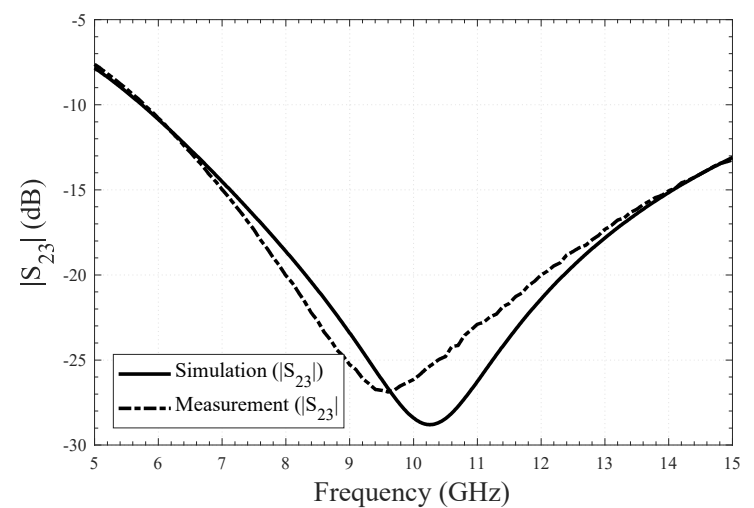

Fig. 11. Simulated and measured values of $\left|\mathrm{S}_{23}\right|$ of divider type-II as a function of frequency.

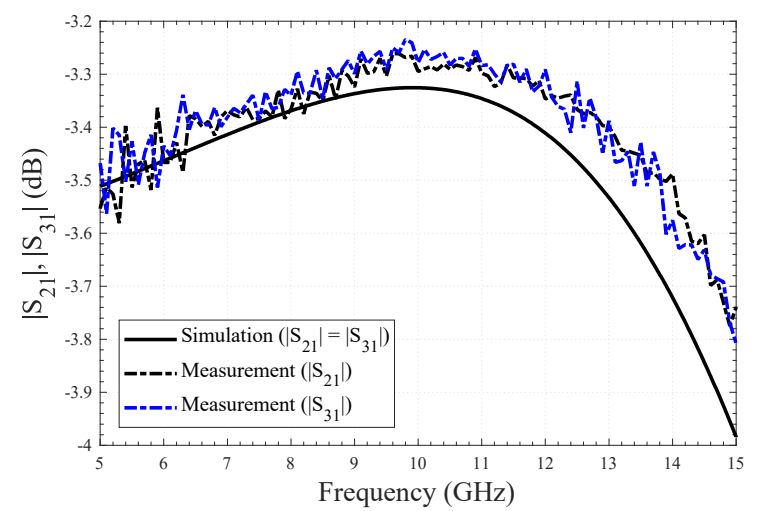

Fig. 12. Simulated and measured values of $\left|\mathrm{S}_{21}\right|$ and $\left|\mathrm{S}_{31}\right|$ of divider type-II as a function of frequency.

\section{CONCLUSION}

Two types of miniaturized lumped element Wilkinson power dividers are proposed, analyzed and design equations are presented. Design examples at $X$-band are fabricated using $\mathrm{GaN}$ on SiC CPW MMIC technology. Divider length reduction up to $72 \%$ is achieved compared to the original Wilkinson power divider without bandwidth degradation thanks to lumped element T-network equivalents, capacitive loading and a symmetrical series $R L C$ circuit in the isolation network. The measured circuits demonstrate at least $20 \mathrm{~dB}$ port matching, at least $20 \mathrm{~dB}$ isolation and at most $0.35 \mathrm{~dB}$ excess insertion loss from $8.9 \mathrm{GHz}$ to $11.9 \mathrm{GHz}$ with a fractional bandwidth of $29 \%$.

\section{ACKNOWLEDGMENT}

The authors would like to thank Bilkent University Nanotechnology Research Center (NANOTAM) for fabricating the power dividers and their support.

\section{REFERENCES}

[1] E. J. Wilkinson, "An N-way hybrid power divider," IRE Trans. Microwave Theory Tech., vol. MTT-8, pp. 116-118, Jan. 1960.

[2] S. B. Cohn, "A class of broadband three-port TEM-mode hybrids," IEEE Trans. Microw. Theory Techn., vol. MTT-16, no. 2, pp. 110-116, Feb. 1968.

[3] J.-C. Kao, Z.-M. Tsai, K.-Y. Lin, and H. Wang, "A modified Wilkinson power divider with isolation bandwidth improvement," IEEE Trans. Microw. Theory Techn., vol. 60, no. 9, pp. 2768-2780, Sep. 2012.

[4] V. Tas and A. Atalar, "An optimized isolation network for the Wilkinson divider," IEEE Trans. Microw. Theory Techn., vol. 62, no. 12, pp. 3393-3402, Dec. 2014.

[5] R. K. Gupta and W. J. Getsinger, "Quasi-lumped-element 3- and 4-port networks for MIC and MMIC applications," in IEEE MTT-S Int. Microwave Symp. Dig., CA, 1984, pp. 409-411.

[6] E. Kaymaksut, Y. Gurbuz, and I. Tekin, "Impedance matching Wilkinson power dividers in $0.35 \mu \mathrm{m}$ SiGe BiCMOS technology," Microwave and Optical Technology Letters, vol. 51, no. 3, pp. 681-685, Mar. 2009.

[7] M. C. Scardeletti, G. E. Ponchak, and T. M. Weller, "Miniaturized Wilkinson power dividers utilizing capacitive loading," IEEE Microw. Wireless Compon. Lett., vol. 12, pp. 6-8, Jan. 2002.

[8] K. Hettak, G. A. Morin, and M. G. Stubbs, "Compact MMIC CPW and asymmetric CPS branch-line couplers and Wilkinson dividers using shunt and series stub loading," IEEE Trans. Microw. Theory Techn., vol. 53, no. 5, pp. 1624-1635, May 2005.

[9] J. Reed and G. Wheeler, "A method of analysis of symmetrical four-port networks," IRE Trans. Microwave Theory Tech., vol. MTT-4, pp. 246-252, Oct. 1956. 